\title{
NEURO-FUZZY MODELLING IN ANAEROBIC WASTEWATER TREATMENT FOR PREDICTION AND CONTROL
}

\author{
Snejana Yordanova ${ }^{1)}$, Rusanka Petrova ${ }^{1)}$, Nelly Noykova ${ }^{2)}$, Plamen Tzvetkov ${ }^{1)}$ \\ ${ }^{1)}$ Faculty of Automation, Technical University of Sofia, 8 Kl.Ohridski blvd., 1000 Sofia, Bulgaria, \\ e-mail: sty@tu-sofia.bg \\ ${ }^{2)}$ Rolf Nevanlinna Institute, Department of Mathematics and Statistics, PO Box 68, FIN-00014, \\ University of Helsinki, Finland, e-mail: nelly.noykova@helsinki.fi
}

\begin{abstract}
The aim of the present paper is to develop neuro-fuzzy prediction models in MATLAB environment of the anaerobic organic digestion process in wastewater treatment from laboratory and simulated experiments accounting for the variable organic load, ambient influence and microorganisms state. The main contributions are determination of significant model parameters via graphical sensitivity analysis, simulation experimentation, design and study of two "black-box" models for the biogas production rate, based on classical feedforward backpropagation and Sugeno fuzzy logic neural networks respectively. The models application is demonstrated in process predictive control.
\end{abstract}

Keywords: Anaerobic digestion of organic waste, neuro-fuzzy modelling, sensitivity, simulation, predictive control

\section{INTRODUCTION}

The anaerobic digestion (methane fermentation) of organic waste is the last stage of water depollution, in which organic matter (animal litters, plant sludge, industrial and domestic waste) is mineralised by microorgamisms in the absence of oxygen to safely disposable in the environment substances. A complementary product is biogas consisting mainly of methane, considered as one of the cleanest non-polluting fuels [1]-[8]. The anaerobic digestion is preferred for the higher organic loads treated, the smaller amount of sludge produced, the energy recovery via utilization of the biogas, the reduced operating costs - no need of oxygen supply and control.

The anaerobic wastewater treatment application is still not very popular because of the process complexity and hence its difficult mathematical description. The models used are nonlinear both in terms of parameters and variables, and nonstationary. The parameter identification encounters various problems [2]-[6] due to the specific features of the microorganisms, low reproducibility of the experiments, limited number of time-consuming and expensive measurements and complex laboratory analyses, noisy experimental data, great number of model parameters, etc.

There arises the need for new types of models of the processes, which describe the nonlinear timevarying behaviour combining knowledge of plant experts, measurements and operational experience. Therefore for the purposes of control, prediction and optimisation fuzzy and neural "black box" models are welcome. The fuzzy sets theory offers a methodology for representing heuristic expert knowledge in a computable way by linguistic labels implemented in linguistic rules thus dealing with uncertainties and avoiding complex mathematical relationships [9], [10]. The fuzzy inference process involves membership functions, fuzzy logic operators and knowledge rules. The membership functions (MFs) allow representation of a degree of membership to a fuzzy set, associated to a linguistic label, for a given input numerical value. The rules ifthen introduce the expert knowledge in a computable way by means mainly of the operators "and" and "or". The fuzzy set and fuzzy logic theory have been successfully applied to different complex process modelling, prediction and control [9] - [12]. The subjectiveness in the choice of the number and the type of MFs as well as their allocation, and in the rule base development can be avoided when inputoutput experimental data is available and an artificial neural network (ANN) trained [11], [12], making use of the basic advantages of multilayer ANNs with nonlinear activation functions to learn from experimental data, to cluster it, to adapt and generalize in mapping nonlinear relationships.

The aim of the recent investigation is to build neuro-fuzzy prediction models of the anaerobic organic digestion process in wastewater treatment on 
the basis of laboratory and simulated experiments accounting for the variable organic load and process parameters in MATLAB environment and to show their application in control.

\section{PROBLEM FORMULATION}

The anaerobic digestion is commonly viewed upon as a three-stage process: hydrolysis and liquefaction of the large insoluble organic molecules; acidogenesis, and methanogenesis [1][4]. The process takes place under prescribed temperature and $\mathrm{pH}$ since the acidogenic bacteria are sensitive to temperature changes while the methanogenic bacteria cannot tolerate $\mathrm{pH}$ fluctuations. In recent years more and more complex mathematical models have been introduced in order to better present the biodegradable processes [1]-[3]. Here the fifth order Hill and Barth-nonlinear model [1] with Monod type specific growth rates $\mu_{1}$ and $\mu_{2}$ of the acidogenic and the methanogenic bacteria respectively is accepted as an average model to fit the data from a number of laboratory experiments in a continuously stirred tank bioreactor with highly concentrated organic pollutants (cattle wastes) at mesophilic temperature [1], [7], [8]. The model describes the multistage process and the diverse groups of involved microorganisms as follows:

$$
\mid \begin{aligned}
& \frac{d S_{o}}{d t}=-D S_{o}-\beta X_{1} S_{o}+D Y_{p} S_{o i} \\
& \frac{d X_{1}}{d t}=\left(\mu_{1}-k_{1}-D\right) X_{1} \\
& \frac{d S_{1}}{d t}=-D S_{1}+\beta X_{1} S_{o}-\frac{\mu_{1} X_{1}}{Y_{1}} \\
& \frac{d X_{2}}{d t}=\left(\mu_{2}-k_{2}-D\right) X_{2} \\
& \frac{d S_{2}}{d t}=-D S_{2}+Y_{b} \mu_{1} X_{1}-\frac{\mu_{2} X_{2}}{Y_{2}} \\
& Q=Y_{g} \mu_{2} X_{2} \\
& \mu_{1}=\frac{\mu_{1 \max } S_{1}}{k_{s 1}+S_{1}}, \mu_{2}=\frac{\mu_{2 \max } S_{2}}{k_{s 2}+S_{2}}
\end{aligned}
$$

where the state space vector $X^{T}=\left[S_{o} X_{1} S_{1} X_{2} S_{2}\right]$ is comprised of the concentrations of: soluble organics $S_{o}, \mathrm{mg} / \mathrm{l}$; acidogenic bacteria $X_{1}, \mathrm{mg} / \mathrm{l}$; substrate for acidogenic bacteria $S_{1}, \mathrm{mg} / \mathrm{l}$; methanogenic bacteria $X_{2}, \mathrm{mg} / \mathrm{l}$; substrate for methanogenic bacteria $S_{2}, \mathrm{mg} / \mathrm{l}$. The only measurable and controlled output is the specific biogas production rate $Q, 1 / 1 . \mathrm{d}, y=[Q], Q(0)=0.04$. The inputs are the dilution rate as control variable $D, \mathrm{~d}^{-1}(D \in[0,0.3])$ and the influent organic concentration as disturbance $S_{o i}, \mathrm{~g} / 1, \quad\left(S_{o i} \in[30,70]\right)$. The vector of the parameters

$$
q^{T}=\left[\beta Y_{p} \mu_{1 \max } k_{s 1} k_{1} Y_{1} \mu_{2 \max } k_{s 2} k_{2} Y_{2} Y_{b} Y_{g}\right]
$$

consists of the coefficients $\beta, \mathrm{d}^{-1}, Y_{p}, \mathrm{mg} / \mathrm{l}, Y_{b}$, $\mathrm{mg} / \mathrm{g}$ and $Y_{g}, 1 / \mathrm{mg}$, the maximal specific growth rate of acidogenic $\mu_{1 \max }, \mathrm{d}^{-1}$ and methanogenic $\mu_{2 \max }, \quad \mathrm{d}^{-1}$ bacteria respectively, the yield coefficients $Y_{1}, \mathrm{mg} / \mathrm{mg}$ and $Y_{2}, \mathrm{mg} / \mathrm{mg}$, the saturation $k_{s 1}, k_{s 2}, \mathrm{mg} / \mathrm{l}$ and the decay $k_{1}, k_{2}, \mathrm{~d}^{-1}$ coefficients for the corresponding bacteria. The initial state vector and the nominal parameter values are respectively:

$$
\begin{aligned}
& X(0)^{o T}=\left[\begin{array}{lllll}
10 & 0.36 & 0.18 & 15.66 & 0.18
\end{array}\right],
\end{aligned}
$$

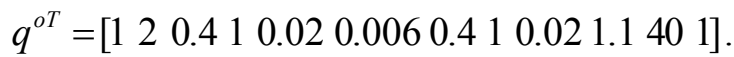

The plant nonlinearity is studied by simulation of model (1). The step responses of the biogas production rate $Q$ to equal incremental step changes of $\Delta 0.05$ of the input $D$ within the range $0-0.3$ when $S_{o i}=50, \mathrm{~g} / 1$ are shown in Fig.1. The influence of the different initial substrate inputs $S_{o i}=30,40,50,60$ is given in Fig. 2 .

The problem is to develop neuro-fuzzy prediction process models using MATLAB accounting for the plant uncertainties due to the change of the operation point along the nonlinear characteristic as a result of variations in the inputs $D$ and $S_{o i}$, in the initial states $X^{\mathrm{T}}(0)$ and in the nominal plant parameters $q^{o \mathrm{~T}}$. These variations reflect the ambient influences, the state of the microorganisms, etc.

The solution of the problem requires the accomplishment of the following tasks:

- graphical sensitivity analysis for determination of the significant sources (initial states $X^{\mathrm{T}}(0)$ and parameters $q^{o \mathrm{~T}}$ ) of dominating influences on the biogas production rate;

- design and carrying out of simulation experiments for collection of realistic inputoutput data accounting for the variations in $D, S_{o i}$ and the significant $X^{\mathrm{T}}(0)$ and $q^{o \mathrm{~T}}$;

- $\quad$ design and training of an ANN model;

- $\quad$ design and training of an ANN, representing Sugeno fuzzy model; model accuracy assessment and comparison between the two models; sample model application for biogas production rate predictive control. 


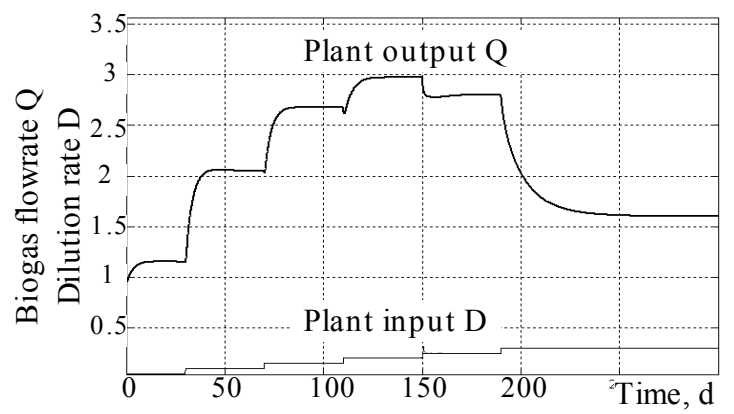

Fig.1 - Sequence of plant step responses

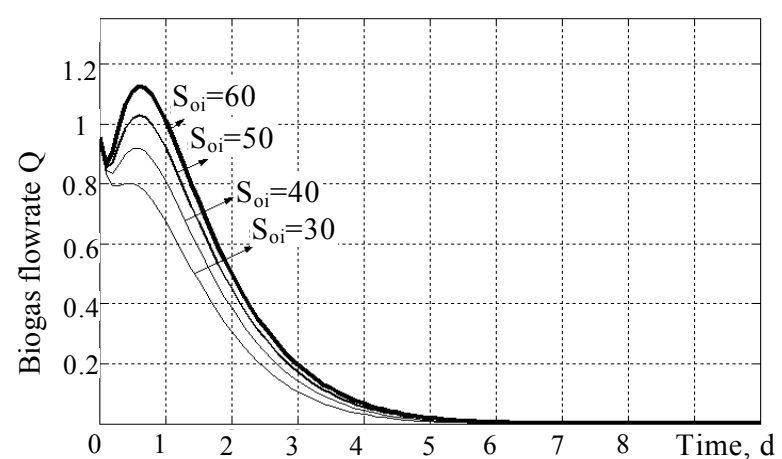

Fig.2 - Plant output $Q$ for different input $S_{o i}$

\section{SENSITIVITY ANALYSIS AND SIMULATION EXPERIMENTATION}

The sensitivity analysis allows excluding correlated and insignificant parameters thus simplifying the plant model. Here it is based on the dimensionless sensitivity functions [3], [4]:

$$
{ }^{T} Q q_{k}(t)=\frac{\partial \ln Q(t)}{\partial \ln q_{k}} \mid q_{k}^{o},{ }^{, T} Q X_{j}(0){ }^{(t)=\left.\frac{\partial \ln Q(t)}{\partial \ln X_{j}(0)}\right|_{X_{j}^{o}(0)}}
$$

The significant simulated sensitivity functions are shown in Fig.3. The sensitivity functions are local properties [4], so the conclusions deduced below by graphical sensitivity analysis depend on the nominal plant parameters and the initial conditions accepted:

1. The significant parameter and initial conditions set is determined for sensitivity functions greater than 1 (in the range of $Q$ ).

2. Parameters and initial conditions with similar influences on $Q$ can be equivalently represented by one of them.

Significant parameters and initial conditions $Y_{p}$ (or $Y_{b}$ ), $X_{1}(0), S_{0}(0)$ and $\beta$ turned out to have similar influence on $Q . Y_{b}$ has been selected as it influences less number of state variables to represent them all in simulation experimentation for providing of realistic input-output data for neuro-fuzzy modelling.
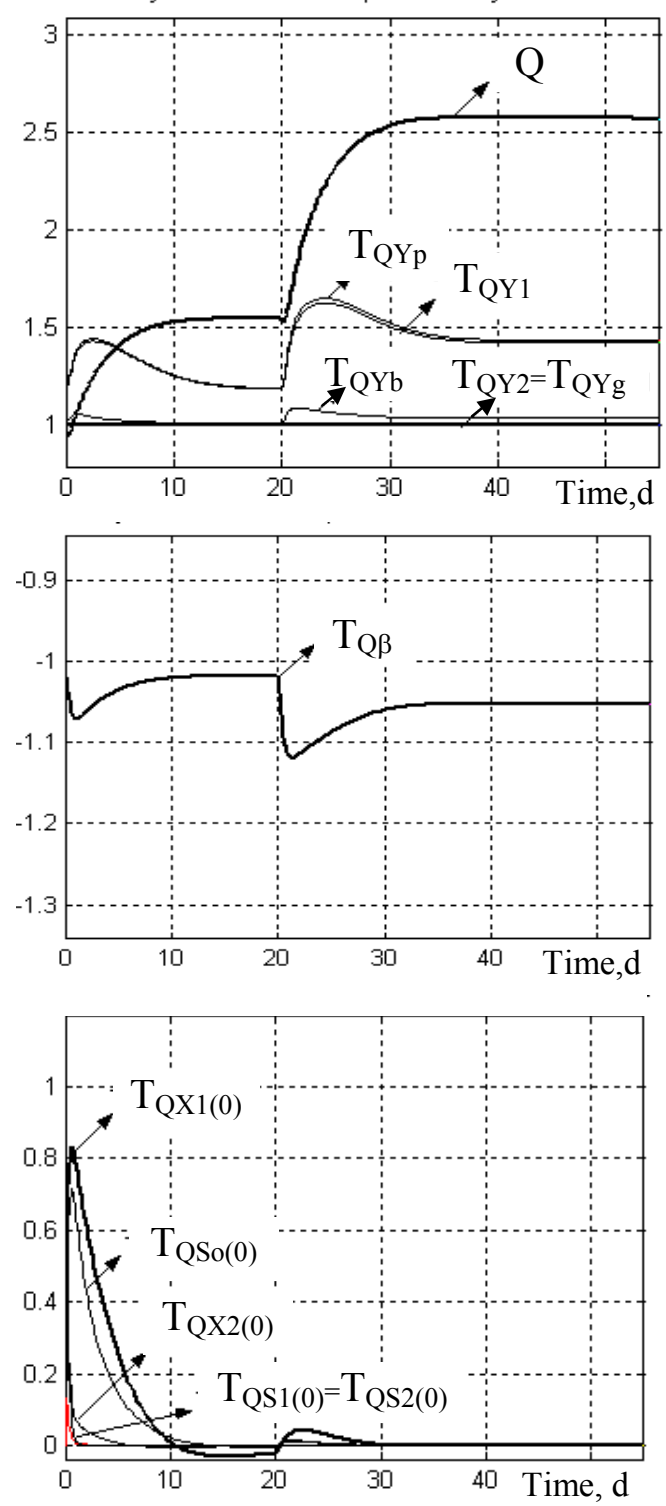

Fig.3 - Sensitivity functions with respect to yield coefficients, kinetic coefficient $\beta$ and initial conditions

The rest of the parameters and of the initial conditions are considered to retain nominal values. Their variations have either negligible impact on the biogas production rate or the impact of their variations can be reflected by variations in $Y_{b}$. Simulation experiments for collecting of data for $Q$ are carried out on a Simulink model based on (1) for $D=0.05 ; 0.1 ; 0.15, S_{\mathrm{oi}}=40 ; 50 ; 60$ for each $D$ and $Y_{\mathrm{b}}=30 ; 40 ; 50$ for fixed $D$ and $S_{\mathrm{o} \text { i }}$. The results are shown in Fig.4.

\section{DESIGN OF NEURAL AND NEURO- FUZZY MODELS}

First, a two-layer feedforward neural network (NN) model for prediction in one time-step ahead 


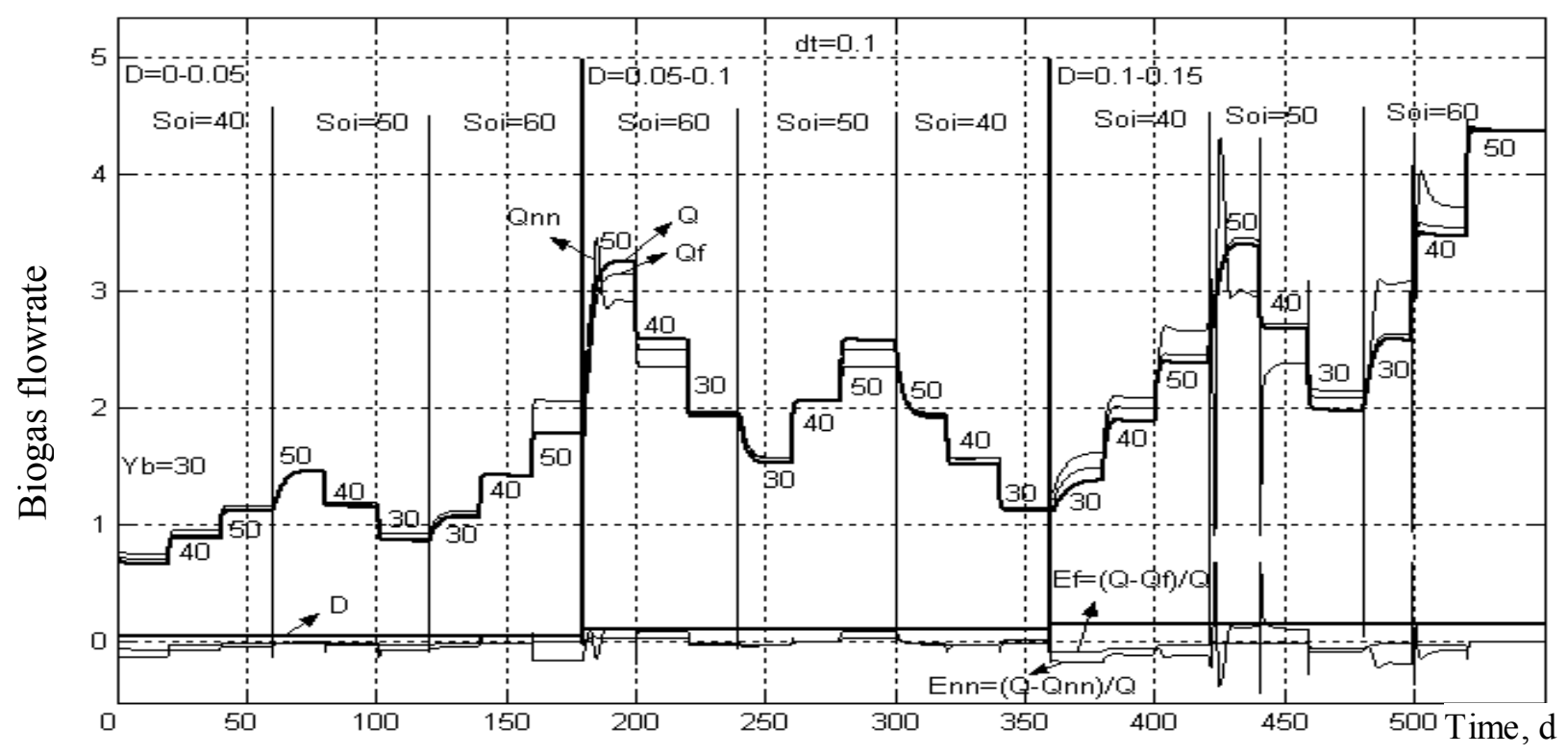

Fig.4 - Simulink simulation experiments using plant model (1), NN and FL plant models

developed. It consists of 7 hidden neurons and logsig activation functions. Its design and training is based on the Neural Networks Toolbox of MATLAB and the backpropagation method. The training data of two inputs $\left[D N_{\mathrm{i}} ; Q N_{\mathrm{i}}\right]$ and one output $Q N_{\mathrm{i}+1}$ is obtained from $Q$ and $D$ from Fig.4, after normalization in the range $[0,1]$.

The final weighting matrices $\mathbf{W}_{1}$ and the bias vectors $\mathbf{B}_{1}$ of the two layers, $l=1,2$, are:

$$
\mathbf{W}_{1}=\left[\begin{array}{cc}
-37.44 & -73.31 \\
-10.22 & -35.25 \\
1.23 & 6.92 \\
10.52 & 35.93 \\
414.36 & -345.28 \\
-10.75 & -37.31 \\
-3.64 & -9.98
\end{array}\right]
$$

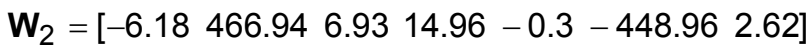
$\mathbf{B}_{1}=\left[\begin{array}{llll}94.12 & 33.17-1.58-31.38-13.8 & 35.083 .44\end{array}\right]$ $\mathbf{B}_{2}=[-1809]$.

Next, in order to simplify the NN model, a Sugeno fuzzy logic (FL) NN model is designed and trained using the same normalized training data and ANFIS (Adaptive Neuro-Fuzzy Inference System) of the Fuzzy Logic Toolbox of MATLAB. For "AND" and "OR" operations are selected the "MIN" and the "MAX" operators, the defuzzyfication method is the weighted average wtaver. After a cluster analysis 3x2 Gaussian MFs for $D N_{\mathrm{i}}$ and $Q N_{\mathrm{i}}$ respectively and 6 rules are initialized. The output functions are linear of the type $Q N_{\mathrm{i}+1}=a \cdot D N_{\mathrm{i}}+b \cdot Q N_{\mathrm{i}}+c$. By training the Sugeno ANN the parameters of the input MFs and the coefficients in the linear outputs of each rule are tuned to reach the final goal -mean squared error below 0.001. The final MFs are shown in Fig.5. The model surface - Fig.6 is highly nonlinear.
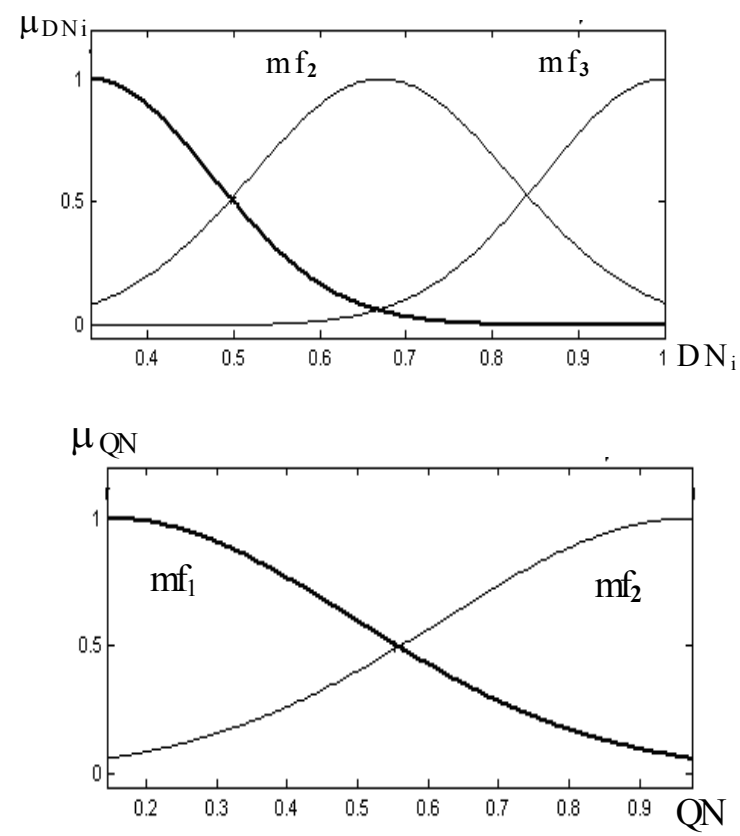

Fig.5 - Membership functions for the input variables DN and QN

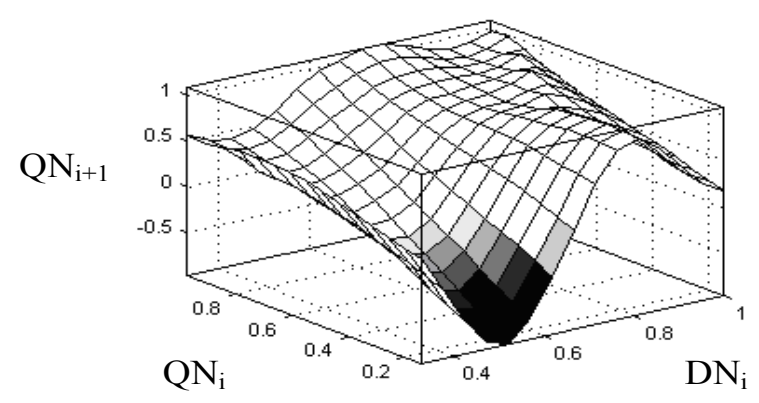

Fig.6 - Input-output model surface 
The tuned rule base is:

1. IF $\mathrm{DN}_{\mathrm{i}}$ is MF1 AND $\mathrm{QN}_{\mathrm{i}}$ is MF1 THEN $\mathrm{QN}_{\mathrm{i}+1}=$ 5.01DN $\mathrm{DN}_{\mathrm{i}}+0.48 \mathrm{QN}_{\mathrm{i}}+2.04$

2. IF $\mathrm{DN}_{\mathrm{i}}$ is MF1 AND $\mathrm{QN}_{\mathrm{i}}$ is MF2 THEN $\mathrm{QN}_{\mathrm{i}+1}=$ 4.65DN $\mathrm{DN}_{\mathrm{i}}-0.92 \mathrm{QN}_{\mathrm{i}}+3.13$

3. IF $\mathrm{DN}_{\mathrm{i}}$ is MF2 AND $\mathrm{QN}_{\mathrm{i}}$ is MF1 THEN $\mathrm{QN}_{\mathrm{i}+1}=$ 13.33DN $\mathrm{DN}_{\mathrm{i}}+1.76 \mathrm{QN}_{\mathrm{i}}-8.74$

4. IF $\mathrm{DN}_{\mathrm{i}}$ is MF2 AND $\mathrm{QN}_{\mathrm{i}}$ is MF2 THEN $\mathrm{QN}_{\mathrm{i}+1}=$ $0.13 \mathrm{DN}_{\mathrm{i}}+1.76 \mathrm{QN}_{\mathrm{i}}-0.47$

5. IF $\mathrm{DN}_{\mathrm{i}}$ is MF3 AND $\mathrm{QN}_{\mathrm{i}}$ is MF1 THEN $\mathrm{QN}_{\mathrm{i}+1}=$ $2.66 \mathrm{DN}_{\mathrm{i}}+1.27 \mathrm{QN} \mathrm{N}_{\mathrm{i}}-3.03$

6. IF $\mathrm{DN}_{\mathrm{i}}$ is MF3 AND $\mathrm{QN}_{\mathrm{i}}$ is MF2 THEN $\mathrm{QN}_{\mathrm{i}+1}=$ $2.27 \mathrm{DN}_{\mathrm{i}}+1.82 \mathrm{QN}_{\mathrm{i}}-3.42$

The simulated responses of the NN model $Q_{\mathrm{nn}}$ and of the neuro-fuzzy model $Q_{\mathrm{f}}$ as well as the relative modelling errors $E_{\mathrm{nn}}$ and $E_{\mathrm{f}}$ are shown in Fig.4. The Sugeno FL NN model is both more simple and accurate as seen from Fig.4.

\section{SAMPLE NEURO-FUZZY MODEL APPLICATION FOR CONTROL}

The organic waste degradation process is difficult to control not only because it is highly nonlinear and non-stationary but also because it is rather slow and unpredictable. The precise control is of crucial importance because the two types of bacteria that perform the digestion are quite sensitive to the environment media. The already trained Sugeno neuro-fuzzy model can be used to predict the next moment plant output knowing the plant input and output in the current moment. It can be embedded in the feedback to supply the controller with advancing information and thus to improve the performance of the modified control system.

A control system configuration with a Sugeno NF controller (NFC), designed in [11], and a Sugeno FL NN plant predictor in the feedback is depicted in Fig.7. The control system error is formed as the difference between the reference for the biogas production rate $Q_{\mathrm{r}}$ and the predicted plant output $Q_{\mathrm{p}}=Q_{\mathrm{i}+1}-e=Q_{\mathrm{r}}-Q_{\mathrm{p}}$. The Sugeno FL NN plant predictor predicts the biogas production rate in the

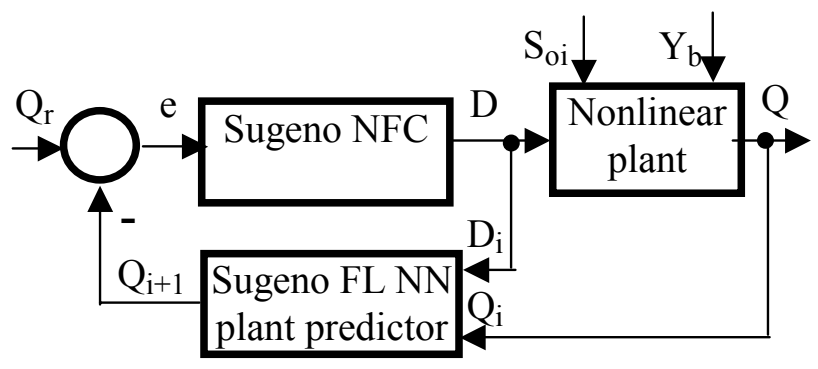

Fig.7 - Block diagram of a control system with Sugeno FL NN plant predictor

next 1 day period on the basis of the current measured plant input $D_{\mathrm{i}}$ and output $Q_{\mathrm{i}}$.
The enclosure of the Sugeno neuro-fuzzy prediction plant model ensures faster transient responses at different operating points, reduces the maximal dynamic deviation and leads to a more smooth, economic and effective control action [11].

\section{CONCLUSIONS}

The main contributions of the paper are the development and comparative study of two "blackbox" prediction models of the biogas production rate in the anaerobic digestion of organic waste in waters, based respectively on classical feedforward backpropagation NN and Sugeno FL NN using MATLAB. The models tackle plant uncertainty related to variable organic loading, ambient influence and microorganisms' state. The FL NN model application in process predictive controller improves the closed-loop system performance.

\section{REFERENCES}

[1] DT. Hill. CL. Barth. A dynamical model for simulation of animal digestion, J. Water Pollution Contr. Fed 10 (1977). p. 2129-2143.

[2] D. Dochain. P. Vanrolleghem. Dynamical Modelling and Estimation in Wastewater Treatment Processes. IWA Publishing, UK, 2001. p. 312

[3] T. G. Müller. N. Noykova. M. Gyllenberg. J. Timmer. Parameter identification in microbial population dynamics models of anaerobic wastewater treatment, J. Math. Biosci. 177-178 (2002). pp.147-160.

[4] N. Noykova. T. G. Müller. M. Gyllenberg. J. Timmer. Quantitative analysis of anaerobic wastewater treatment processes: identifiability and parameter estimation, J. Biotech. Bioeng., 78 (1) (2002). pp. 89-103.

[5] I. Simeonov. V. Momchev. D. Grancharov. Dynamic modeling of mesophilic anaerobic digestion of animal waste, J. Wat. Res. 30 (5) (1996). pp. 1087-1094.

[6] I. Simeonov. Modeling and control of anaerobic digestion of organic waste, J. Chem. Biochem. Eng. Q. 8 (2) (1994). pp. 42-52.

[7] O. Z. Bernard. D. Dochain. Advanced monitoring and control of anaerobic wastewater treatment plants: II - dynamic model development and identification. Proceedings of the $5^{\text {th }}$ Int. Symp. WATERMATEX, Gent, Belgium, September 2000. pp. 3.57-3.64.

[8] S. Yordanova. N. Noikova. Influence of perturbations on the waste water treatment process, J. Chem. Biochem. Eng. Q. 10 (1) (1996). pp. 9-14. 
[9] T. J. Ross. Fuzzy Logic With Engineering Applications. McGraw Hill, Inc., 1995.

[10] J.-S.R. Jang. C.-T. Sun. E. Mizutani. NeuroFuzzy and Soft Computing. A Computational Approach to Learning and Machine Intelligence. MATLAB Curriculum Series. Prentice-Hall, Inc., N.J., 1997. ISBN 0-13-261066-3.

[11] S. Yordanova. Fuzzy two-level control for anaerobic wastewater treatment. Proceedings of the 2-nd Int. IEEE Conf. "Intelligent Systems", Varna, June 22-24, Sofia, Bulgaria 2004, pp. 348-352.

[12] S. Yordanova. R. Petrova. V. Mladenov. Neuro-fuzzy control for anaerobic wastewater treatment, WSEAS Trans. on Systems (3) (2004). pp. 724-129, ISSN 1109-2777

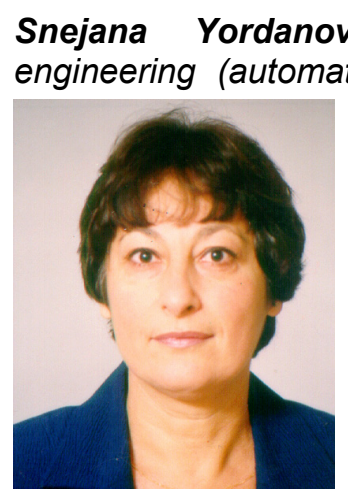

- MEng in electrical ic control) (1977) and Ph.D. (1986) both from the Technical University of Sofia (TUS), Bulgaria. She is a fulltime Assoc. Prof. with the Department of Process Control, Faculty of Automation, TUS. E-mail: sty@tu-sofia.bg. Scientific and research interests: robust, fuzzy logic and neural network approaches to system modelling, simulation and control in oil refining and milk processing industries, wastewater treatment; measurement systems; thermal power plants. Publications - over 100, textbooks -5 , manuals -2 , research projects 14.

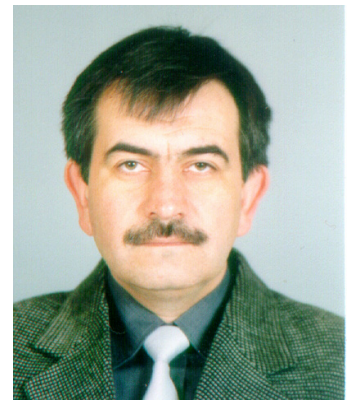

Plamen Tzvetkov - MEng in electrical engineering (electric measurements) (1985) and Ph.D. (1998) both from the Technical University of Sofia (TUS), Bulgaria. He is a full-time Assoc. Professor with the Department of Electrical Measurements, Faculty of Automation, TUS. E-mail: tzvetkov@tu-sofia.bg. Scientific and research interests: robust measurement systems, intelligent measurement systems, computer hardware and software for temperature and magnetic characteristics measurement. Publications - over 40, textbooks - 2, manuals -6, research projects 11 .
Nelly Noykova - M.Sc. in bioengineering (Automation of Biotechnological Processes) (1991)

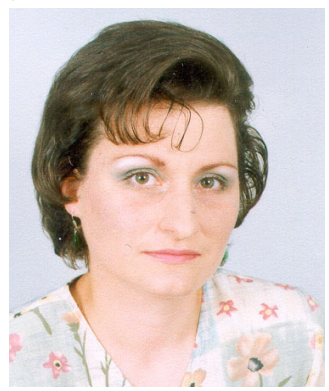
from the Technical University of Sofia (TUS), Bulgaria, and Ph.D. (2005) from the University of Turku, Finland. She is a researcher in the Group of Biomathematics and Biometry, Department of Mathematics and Statistics, University of Helsinki, Finland. E-mail: nelly.noykova@helsinki.fi Scientific and research interests: biological wastewater treatment, theoretical and practical identifiability in nonlinear ODEs models, sensitivity theory, parameter estimation, genotype $x$ - expression interactions, Bayesian models. Publications - over 10, manuals 1 , research projects 2 .

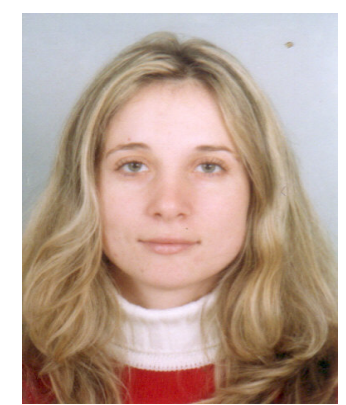

Rusanka Petrova - MEng in electrical engineering (automatic control) (2004) from the Technical University of Sofia (TUS), Bulgaria. She is a Ph. D. student (2004) with the Department of Process Control, Faculty of Automation, TUS. E-mail: rusanka_petrova@yahoo.co m. Scientific and research interests: robust, fuzzy logic and neural network approaches for system modelling, biological wastewater treatment. Publications - 4. 